\title{
Editorial
}

\section{Good winds are expected!}

\author{
Carlos Renato Zacharias \\ Editor-in-Chief, International Journal of High Dilution Research (IJHDR)
}

Probably by cultural and historical reasons, Western Europe occupied the center of homeopathy research stage. It was in Western Europe that Hahnemann initially established the grounds of homeopathy, and also were Western European the researchers who have been trying to characterize the scientific bases behind high dilutions biological action ever since. Europe witnessed all phases of homeopathy development, its growth and also its decline, its time of glory as well as its many crises. Ideological divergences - sometimes grounded on irresponsible attitudes by homeopaths themselves, sometimes arising from skeptics pride and prejudice gave rise to political and social movements against homeopathy. In spite of this, clinical and experimental evidences kept homeopathy alive as an important therapeutic option able to reunite low cost and efficacy provided its conceptual basis and limitations are observed.

There is basic agreement among all involved in homeopathy and high dilutions - either friends or skeptics - on that the science behind them is still hidden, they still wait for a sound theory able to explain their basic mechanisms, different features and experimental evidences. For this reason, high dilution research is a significant scientific enterprise with potential social, politic, economic, environmental and scientific effects.

High-quality and innovative science demands funding, a critical mass, institutional references, thorough discussions and a quiet and open intellectual context to develop new theories and experimental models. For many years, Western Europe was the place that supplied these conditions. However, it has also been the place where the strongest criticism and barriers against application and development of HD research were risen up.

While Western Europe was engaged in these matters, new research poles appeared and shifted discussion and laboratory and clinical work from Western to Eastern Europe, India and Brazil, among other areas of the world. This shift is patent in the increasing amount of scientific articles published in peer-reviewed journals originated in these new areas, the organization of scientific meetings and the active participation of students and young researchers in these activities.

More than a fad, high dilutions research represents an attempt to keep the true and open-minded scientific spirit alive. A new generation of researchers has emerged to populate spaces left empty. The time is ripe for emergent research groups to compete on equal footing with the more traditional ones, since in the end all of us are looking for new notions, new models and new theoretical foundations.

A recent example was given by the XXV GIRI Symposium (GIRI - Groupe International de Recherche sur l'Infinitésimal) held at Foz do Iguaçu (Brazil) on September 4-7 2011. The number of students and young researchers was remarkable, and thematic workshops and more than 50 studies covering the many-fold features of HD research were presented and discussed.

This issue of IJHDR is fully devoted to XXV GIRI Symposium and includes a summary of presentations ranging from basic to clinical research, with an emphasis on the biological side - in vitro and in vivo; cellular, animal and vegetal models. Therefore, this issue introduces new HD researchers and research-groups to the scientific community. 
A new generation is set in motion and both IJHDR and GIRI are essential institutional references to support and give direction to this movement. GIRI is the natural locus where debate must converge in order for researchers to be able to dialogue and produce knowledge. In turn, IJHDR is a modern and dynamic publishing medium fit to divulgate knowledge thus produced.

More than ever, HD research appears as an emergent and highly active field! And much work still needs to be done. The academic geography of HD research is changing. It is not a matter of replacing old by new research centers. As a fact, HD research is expanding its boundaries, its scientific community has started sharing responsibility and joining efforts. As any other scientific field, also HD research is building a critical mass, which is a sine qua non requirement for research to attain the quality demanded by contemporary science.

New winds are blowing and they will surprise those little prepared or unexpecting.

\section{(cc) BY-NC-ND Licensed to GIRI.}

How to cite this article: Zacharias CR. Good winds are expected [editorial]. Int J High Dilution Res [online]. 2011 [cited YYYY Month dd]; 10(36):95:96. Available from: http://www.feg.unesp.br/ ojs/index.php/ijhdr/article/view/524/537 